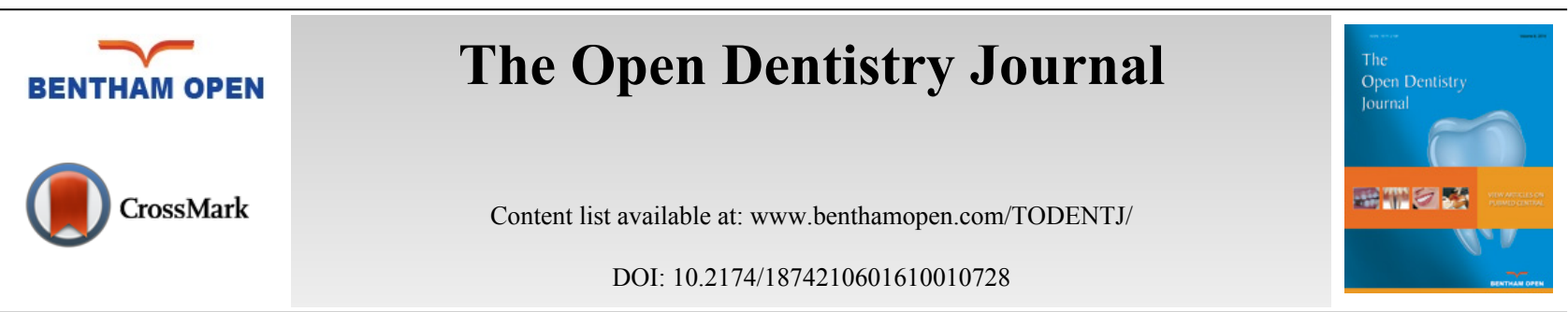

RESEARCH ARTICLE

\title{
Temporal Evaluation of Neurosensory Complications After Mandibular Third Molar Extraction: Current Problems for Diagnosis and Treatment
}

\author{
Masaya Akashi", Yujiro Hiraoka, Takumi Hasegawa and Takahide Komori \\ Department of Oral and Maxillofacial Surgery, Kobe University Graduate School of Medicine, Kusunoki-cho 7-5-1, \\ Chuo-ku, Kobe 650-0017, Japan
}

Received: June 30, 2016

Revised: November 07, 2016

Accepted: December 08, 2016

\begin{abstract}
:
Objective:

This retrospective study aimed to report the incidence of neurosensory complications after third molar extraction and also to identify current problems and discuss appropriate management of these complications.
\end{abstract}

\section{Method:}

Patients who underwent extraction of deeply impacted mandibular third molars under general anesthesia were included. The following epidemiological data were retrospectively gathered from medical charts: type of neurosensory complication, treatment for complication, and outcome.

\section{Results:}

A total 369 mandibular third molars were extracted in 210 patients under general anesthesia during this study period. Thirty-one of the 369 teeth $(8.4 \%)$ in 31 patients had neurosensory complications during the first postoperative week resulting from inferior alveolar nerve damage. Neurosensory complications lasting from 1 to 3 months postoperatively included 17 cases of hypoesthesia and 8 of dysesthesia in 19 patients. Five cases of hypoesthesia and 4 of dysesthesia in 5 patients persisted over 1 year postoperatively. Sixteen of 369 teeth $(4.3 \%)$ in 16 patients had persistent neurosensory complications after third molar extraction under general anesthesia. Stellate ganglion block was performed in 4 patients. Early initiation of stellate ganglion block (within 2 weeks postoperatively) produced better outcomes than late stellate ganglion block (over 6 months postoperatively).

\section{Conclusion:}

Refractory neurosensory complications after third molar extraction often combine both hypoesthesia and dysesthesia. Current problems in diagnosis and treatment included delayed detection of dysesthesia and the lack of uniform timing of stellate ganglion block. In the future, routinely inquiring about dysesthesia and promptly providing affected patients with information about stellate ganglion block might produce better outcomes.

Keywords: Third molar extraction, Neurosensory complication, Medication, Stellate ganglion block, Hypoesthesia, Dysesthesia.

\section{INTRODUCTION}

Although the common complications after third molar extraction were pain and swelling, the most serious one is the neurosensory complication which occurs with an incidence of 0.3 to $8.4 \%$ [1,2]. In our previous retrospective study of surgical extraction of 440 mandibular third molars, the incidence of inferior alveolar nerve (IAN) injury at 1 month after surgery was $6.4 \%$ [3]. Risk factors included panoramic radiographic signs, such as loss of the white line of the inferior

\footnotetext{
* Address correspondence to this author at the Department of Oral and Maxillofacial Surgery, Kobe University Graduate School of Medicine, Kusunoki-cho 7-5-1, Chuo-ku, Kobe 650-0017, Japan; Tel: +81-78-382-6213; Fax: +81-78-382-6229, E-mail: akashim@med.kobe-u.ac.jp
} 
alveolar canal or diversion of the canal, and excessive hemorrhage during extraction [3]. Although numerous studies have analyzed the incidence and risk factors of post-extraction neurosensory complications, few have reported treatments for complications [2, 4], probably because of clinical challenges. A recent study reported that stellate ganglion block (SGB) could be an effective method for treating neurosensory deficits of IAN following orthognathic surgery [5].

One such challenge is the lack of uniformity of sensory testing in patients with post-extraction complications, which makes comparing data impossible and highlights the necessity of uniform testing methodology [6]. The difficulty of accurate differentiation of sensory nerve impairment (i.e., paresthesia, dysesthesia, hypoesthesia, anesthesia, and pain $[3,4]$ ) is also often problematic. Moreover, there is insufficient evidence concerning the efficacy of different treatment modalities for neurosensory complications after third molar extraction [2].

The purpose of this retrospective study was to report the incidence of persistent neurosensory complications after third molar extraction under general anesthesia in our hospital and to evaluate the temporal changes in neurosensory complications. We also aimed to identify current problems in the diagnosis and treatment of neurosensory complications after mandibular third molar extraction, and to discuss the appropriate management of these problematic complications.

\section{MATERIALS AND METHODS}

This retrospective study included patients who underwent surgical removal of impacted mandibular third molars only under general anesthesia in our department from September 2011 to April 2015. Before surgery, computed tomography (CT) examination was performed in all cases. In mandibular third molars included in this study, the roots were superimposed, or impinged to the mandibular canal, or the distances between the roots and the canals were less than $2 \mathrm{~mm}$ on CT images, namely, the classification of the relationship of the roots to the IAN on CT images introduced in our previous study [3] was types 1, 2, 3, or 4. These inclusion criteria are reasonable for the temporal evaluation of post-extraction complications, because our surgeons perform routine follow-up after difficult procedures which require general anesthesia.

The surgical method is described in detail in our previous report [3]. In brief, triangular mucoperiosteal flap was raised, followed by vestibular bone removal. If necessary, crown and root sectioning were performed with fissure burs. Sockets were irrigated with sterile saline solution. A multifilament absorbable suture was used to close the wound. Surgeons in this study included senior residents and specialists in our department. Antibiotic and anti-inflammatory agents were administrated postoperatively as follows: intravenous cefmetazole sodium, $2 \mathrm{~g}$ daily for 3 days; intravenous hydrocortisone sodium succinate, $200 \mathrm{mg}$ at wound closure; and oral loxoprofen sodium, $180 \mathrm{mg}$ daily.

The following epidemiological data were retrospectively gathered from the medical charts: age, sex, sensory test performed, type of neurosensory complication, treatment for complication, and treatment outcome. Neurosensory complications were diagnosed by each attending doctor and were monitored for 7, 14, or 30 days, or longer if necessary. Complications were retrospectively classified as hypoesthesia (diminished sensation), anesthesia (absence of sensation), or dysesthesia (painful sensation triggered by non-noxious stimuli) [3], based on medical charts. Attending doctors determined the treatment for neurosensory complications. Some patients were referred to the Division of Anesthesiology at our hospital. Anesthesiologists performed SGB and prescribed drugs, with the exception of vitamin B12 (VB12) and adenosine triphosphate (ATP). This was a nonrandomized, retrospective study of patients and was thus granted exemption from institutional review board approval by our institution.

\section{RESULTS}

A total of 369 mandibular third molars was extracted under general anesthesia in 210 patients during this study period. Thirty-one of 369 teeth (8.4\%) in 31 patients (12 male and 19 female) had various neurosensory complications within 1 week after surgery. All complications resulted from IAN lesions. None of the patients experienced postoperative anesthesia. Medical inquiry about mentum and lip sensitivity, pin-prick, and light touch test were performed in all 31 patients.

Temporal changes in neurosensory complications are shown in Fig. (1a). Neurosensory complications persisting from 1 to 3 months after surgery included 17 cases of hypoesthesia and 8 of dysesthesia in 19 patients. Neurosensory complications lasting more than 1 year after surgery included 5 cases of hypoesthesia and 4 of dysesthesia in 5 patients. 
$\mathbf{a}$



b

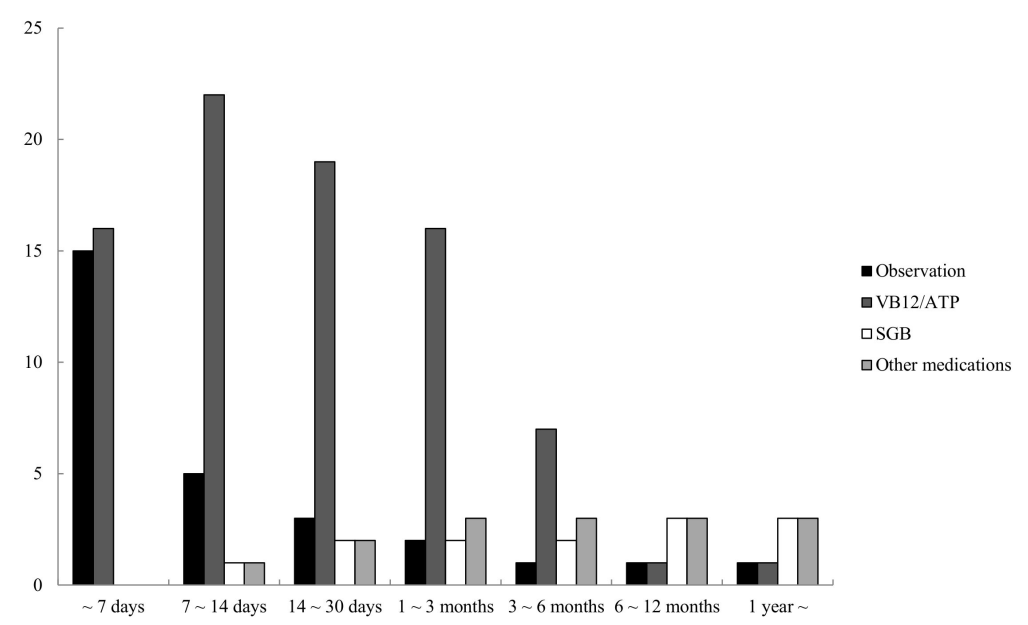

Fig. (1). (a) Temporal changes in neurosensory complications. Some patients had multiple symptoms. (b) Temporal changes in treatments. Some patients received multiple treatments. VB12, Vitamin B12; ATP, adenosine triphosphate; SGB, stellate ganglion block.

The temporal changes in treatments for neurosensory complications are shown in Fig. (1b). Treatments administered within 1 week after extraction included oral administration of both VB12 and ATP in 16 patients; 15 patients were simply observed. SGB was performed in 4 patients: 1 patient received treatment from 1 week to 6 months after extraction, 1 patient from 2 weeks to 1 year, and 2 patients from 6 months to 1 year. There were no complications of SGB. Four patients received medications other than VB12 and ATP, including anticonvulsant (pregabalin), serotonin-noradrenalin reuptake inhibitor (duloxetine), and neurotropin ${ }^{\circledR}$.

The median follow-up duration was 2 months (range, 1-36 months). Fifteen of 31 patients (48.4\%) achieved resolution of neurosensory complications during the follow-up period. The overall incidence of persistent neurosensory complications after third molar extraction under general anesthesia was 16 of 369 teeth (4.3\%) in 16 patients. Eleven of 16 patients discontinued follow-up and treatments for neurosensory complications because their symptom was only hypoesthesia, which was not responding to treatment and did not affect their daily life. Of 5 patients with neurosensory complications persisting over 1 year after surgery, 2 achieved amelioration of hypoesthesia or dysesthesia. One of these 2 patients received SGB from 1 week to 6 months and the other from 2 weeks to 1 year after extraction. In contrast, both patients who received SGB from 6 months to 1 year after extraction experienced persistent hypoesthesia and dysesthesia. 


\section{DISCUSSION}

The incidence of persistent neurosensory complications after third molar extraction under general anesthesia in this study might be relatively high $(4.3 \%)$, because the roots of extracted third molars included in this study exhibited direct contact or close proximity to the mandibular canal on CT images. Dysesthesia was more likely to be persistent than other complications. This result indicates the importance of early detection of dysesthesia and immediate initiation of treatment for it. We also found clinical challenges through this retrospective study.

Various methods of sensory testing have been described in previous studies [4, $6-8]$. Poort et al. recommend the light touch test with Semmes-Weinstein monofilaments, which is reproducible but time-consuming, and also note the importance of patients' subjective evaluation of neurosensory deficits [6]. In general, neurosensory deficits after third molar extraction spontaneously recover within 6 months after surgery [9]; in this study, almost all cases of hypoesthesia recovered within 6 months. As shown in Fig. (1a), dysesthesia was not detected in some cases within the first month after surgery. Hypoesthesia sometimes develops dysesthesia, and careful attention is needed especially in cases developing dysesthesia [4]. Repair reaction of nerve damage following IAN injury is completed within 2 to 3 weeks [10]. During this period, adjacent nerve fibers may be accidentally connected, subsequently, impulses from the peripheral nerves may be transmitted to the wrong destination [10]. Inappropriate nerve fiber connection (so-called "ephapse") often results in neuroma formation, which exhibit spontaneous discharge (i.e., dysesthesia) [10]. If dysesthesia develops during recovery period, the prognosis will be poor in contrast to hypoesthesia alone [10]. We referred patients with problematic neurosensory complications after third molar extraction to anesthesiologists (pain specialists) at our hospital. In fact, these clinicians detected dysesthesia in some cases. To overcome the problem of overlooking dysesthesia, attending surgeons should routinely ask patients about symptoms of dysesthesia (e.g., Do you have "tingling", "burning", "prickling", or "itchiness" of the mentum or lip?) [4]. Patients with neurosensory complications that do not harm quality of life sometimes discontinue follow-up care. It would be better to use a visual analog scale-based questionnaire for evaluating patients' subjective sensitivity, as recommended by Poort et al. [6].

A second problem in managing neurosensory complications is the lack of the standardization of treatments, especially the optimal timing of SGB. It has been recently reported that SGB may be an effective treatment for neurosensory deficits after orthognathic surgery [5]. SGB increases tissue blood flow in the head, face, and neck, because of its sympatholytic effects [11]. An experimental study in rabbits showed that blood flow in the common carotid artery, tongue mucosa, mandibular bone marrow, and masseter muscle on the block side increased after SGB [12]. The potential complications of SGB include technical complications (injury of the brachial plexus, trachea, esophagus, pleura, or lung), pharmacologic complications (hoarseness and local anesthetic toxicity), and infection [5]. In a recent report, SGB was used for IAN paresthesia after retromolar bone harvesting for alveolar ridge augmentation before dental implant insertion. That study found that SGB accelerated recovery from postoperative neurosensory disturbances [13]. Although our study included only 4 patients treated with SGB, neurosensory symptoms ameliorated in those patients in whom SGB was initiated from 1 to 2 weeks after extraction, whereas both hypoesthesia and dysesthesia persisted in patients in whom SGB was initiated over 6 months postoperatively. In a previous report, acupuncture was performed for anesthesia and hypoesthesia resulting from lingual or inferior alveolar nerve injury [14]. Treatment outcomes in that study were more favorable when acupuncture treatment was initiated within 18 months after nerve injury. Although there is no evidence of a correlation between outcomes and the timing of SGB, many physicians believe that earlier treatment produces better outcomes. In a study by Nogami et al. [13], VB12 was administered in patients with neurosensory disturbances 1 week postoperatively, with a maximum medication period of 6 months; if there was no amelioration of symptoms, SGB was performed. Oral and maxillofacial surgeons should at least provide information about SGB if symptoms persist 2 weeks after extraction, especially in patients with dysesthesia.

\section{CONCLUSION}

In conclusion, the incidence of persistent neurosensory complications after third molar extraction under general anesthesia in this study was $4.3 \%$. Refractory neurosensory complications often combined both hypoesthesia and dysesthesia. The early detection of dysesthesia is important. In the future, medical inquiry about dysesthesia should be routinely performed after third molar extraction. In patients with post-extraction neurosensory complications, especially dysesthesia, information about SGB should be provided as early as possible.

\section{CONFLICT OF INTEREST}

The authors indicate full freedom of investigation and no potential conflict of interest. 


\title{
ACKNOWLEDGEMENTS
}

\author{
Declared none.
}

\section{REFERENCES}

[1] Jerjes W, Swinson B, Moles DR, et al. Permanent sensory nerve impairment following third molar surgery: a prospective study. Oral Surg Oral Med Oral Pathol Oral Radiol Endod 2006; 102(4): e1-7. [http://dx.doi.org/10.1016/j.tripleo.2006.01.016] [PMID: 16997083]

[2] Leung YY, Fung PP, Cheung LK. Treatment modalities of neurosensory deficit after lower third molar surgery: a systematic review. J Oral Maxillofac Surg 2012; 70(4): 768-78. [http://dx.doi.org/10.1016/j.joms.2011.08.032] [PMID: 22177820]

[3] Hasegawa T, Ri S, Shigeta T, et al. Risk factors associated with inferior alveolar nerve injury after extraction of the mandibular third molara comparative study of preoperative images by panoramic radiography and computed tomography. Int J Oral Maxillofac Surg 2013; 42(7): 843-51. [http://dx.doi.org/10.1016/j.ijom.2013.01.023] [PMID: 23499150]

[4] Mahon N, Stassen LF. Post-extraction inferior alveolar nerve neurosensory disturbancesa guide to their evaluation and practical management. J Ir Dent Assoc 2014; 60(5): 241-50. [PMID: 25638921]

[5] Nogami K, Taniguchi S. Stellate ganglion block, compared with xenon light irradiation, is a more effective treatment of neurosensory deficits resulting from orthognathic surgery, as measured by current perception threshold. J Oral Maxillofac Surg 2015; 73(7): 1267-74. [http://dx.doi.org/10.1016/j.joms.2015.01.012] [PMID: 25900233]

[6] Poort LJ, van Neck JW, van der Wal KG. Sensory testing of inferior alveolar nerve injuries: a review of methods used in prospective studies. J Oral Maxillofac Surg 2009; 67(2): 292-300. [http://dx.doi.org/10.1016/j.joms.2008.06.076] [PMID: 19138602]

[7] Bhat P, Cariappa KM. Inferior alveolar nerve deficits and recovery following surgical removal of impacted mandibular third molars. J Maxillofac Oral Surg 2012; 11(3): 304-8.

[http://dx.doi.org/10.1007/s12663-012-0335-0] [PMID: 23997481]

[8] Sarikov R, Juodzbalys G. Inferior alveolar nerve injury after mandibular third molar extraction: a literature review. J Oral Maxillofac Res 2014; 5(4): e1. [http://dx.doi.org/10.5037/jomr.2014.5401] [PMID: 25635208]

[9] Cheung LK, Leung YY, Chow LK, Wong MC, Chan EK, Fok YH. Incidence of neurosensory deficits and recovery after lower third molar surgery: a prospective clinical study of 4338 cases. Int J Oral Maxillofac Surg 2010; 39(4): 320-6. [http://dx.doi.org/10.1016/j.ijom.2009.11.010] [PMID: 20061121]

[10] Fukuda K, Ichinohe T, Kaneko Y. Pain management for nerve injury following dental implant surgery at tokyo dental college hospital. Int J Dent 2012; 2012: 9 .

[http://dx.doi.org/10.1155/2012/209474]

[11] Melis M, Zawawi K, al-Badawi E, Lobo Lobo S, Mehta N. Complex regional pain syndrome in the head and neck: a review of the literature. J Orofac Pain 2002; 16(2): 93-104. [PMID: 12043524]

[12] Terakawa Y, Ichinohe T, Kaneko Y. Redistribution of tissue blood flow after stellate ganglion block in the rabbit. Reg Anesth Pain Med 2009; 34(6): 553-6.

[http://dx.doi.org/10.1097/AAP.0b013e3181b4c505] [PMID: 19916209]

[13] Nogami S, Yamauchi K, Shiiba S, Kataoka Y, Hirayama B, Takahashi T. Evaluation of the treatment modalities for neurosensory disturbances of the inferior alveolar nerve following retromolar bone harvesting for bone augmentation. Pain Med 2015; 16(3): 501-12. [http://dx.doi.org/10.1111/pme.12618] [PMID: 25533572]

[14] Ka L, Hirata Y, Kobayashi A, Wake H, Kino K, Amagasa T. Treatment results of acupuncture in inferior alveolar and lingual nerves sensory paralysis after oral surgery. Kokubyo Gakkai Zasshi 2006; 73(1): 40-6. [http://dx.doi.org/10.5357/koubyou.73.40] [PMID: 16629469]

(C) Akashi et al.; Licensee Bentham Open

This is an open access article licensed under the terms of the Creative Commons Attribution-Non-Commercial 4.0 International Public License (CC BY-NC 4.0) (https://creativecommons.org/licenses/by-nc/4.0/legalcode), which permits unrestricted, non-commercial use, distribution and reproduction in any medium, provided the work is properly cited. 